\title{
Efeito da bandagem elástica em testes funcionais e na co-contração dos músculos do tornozelo em praticantes de Crossfit
}

https://doi.org/10.11606/issn.1981-4690.v35i2p272-281

\section{Resumo}

0 uso da bandagem elástica pode gerar efeitos positivos sobre o controle neuromuscular e biomecânica do movimento, tal como o aumento da estabilidade articular. Assim, o objetivo do estudo foi avaliar o efeito da bandagem elástica na co-contração dos músculos do tornozelo, na cinemática e no desempenho durante testes funcionais em jovens praticantes de Crossfit ${ }^{\circledR}$. Participaram do estudo 21 jovens (idade: $27,1 \pm 5,8$ anos) praticantes de Crossfit ${ }^{\circledR}$. No primeiro dia de coleta foram realizados os procedimentos: as contrações isométricas voluntárias máximas de gastrocnêmio medial e tibial anterior, familiarização e coleta de dados do salto vertical. No segundo dia, foram realizados: a coleta de dados dos testes funcionais (Multiple Hop Test - MHT; e Star Execution Balance Test - SEBT). Todos os testes foram realizados nas condições com e sem bandagem elástica, com a ordem aleatorizada e um intervalo de cinco minutos entre as condições. Para a análise cinemática foi considerado a aceleração do tornozelo nos eixos: transversal $(X)$ e horizontal (Z), durante a aterrissagem do salto. 0 nivel de significância foi de $p<0,05.0$ ANOVA one-way medidas repetidas não encontrou diferença para as comparações realizadas de co-contração e cinemática. Durante o SEBT, houve aumento do alcance máximo de 3,8\% e 5,6\% nas direções ântero medial $(p=0,001)$ e póstero medial $(p=0,005)$ durante a condição com bandagem elástica. No MHT, houve redução do tempo de execução, em 5,7\% e 7,1\% dos saltos em progressão anterior com desafios $(p=0,018$ e $p=0,009)$ na condição com bandagem elástica. 0 uso da bandagem elástica melhorou o desempenho em testes funcionais de jovens praticantes de $\mathrm{Crossfit}^{\circledR}$, possivelmente, decorrente de efeito placebo.

Palavras-chave: Cinemática; Eletromiografia; Fisioterapia.

\section{Introdução}

A bandagem elástica é uma técnica terapêutica que foi criada por Kenzo Kase ${ }^{1}$. Esta técnica se diferencia de bandagens rígidas, pelo fato de que é possível alongar a fita, além do comprimento original, o que possibilita uma menor restrição mecânica ao movimento ${ }^{2}$. A tensão mecânica criada pela fita estirada sobre a pele resulta na estimulaçấo dos mecanoreceptores, que podem gerar efeitos táteis e mecânicos ${ }^{3}$.

O uso da bandagem elástica tem sido amplamente difundido no meio esportivo, como forma de prevenção de lesôes, bem como, recurso auxiliar no processo de reabilitação ${ }^{4,5}$. Estudo pregresso identificou o efeito positivo do uso da bandagem elástica no aumento da ativação eletromiográfica dos músculos estabilizadores da escapula em atletas de baseball com síndrome do impacto de ombro ${ }^{4}$. Além disso, também foi identificado uma redução do momento vertical da força de reaçáo do solo (FRS) e amplitude de movimento de inversão do tornozelo, durante o salto máximo em atletas com instabilidade funcional de tornozelo (IFT) 5 .

Em contrapartida, HuANG et al. ${ }^{6}$ demonstrou que o uso da bandagem elástica aumentou o momento vertical da FRS durante o salto máximo, em relação a condição placebo, o que representa uma alteração 
biomecânica que pode resultar em maior risco de entorses. Neste mesmo estudo, apesar da desvantagem mecânica gerada pela bandagem elástica, esses autores também identificaram um aumento da ativação do músculo gastrocnêmio medial, o que poderia resultar em aumento da potência muscular durante o salto ${ }^{6}$. Porém, diferente do esperado, estes mesmos autores não encontraram melhora no desempenho do salto (altura) com o uso da bandagem elástica. Nesse sentido, os efeitos da bandagem elástica sobre o sistema neuromuscular e na biomecânica de gestos desportivos ainda são controversos.

Apesar de ainda não ser bem esclarecido o efeito da bandagem elástica no controle neuromuscular e na biomecânica, estudos anteriores relataram melhora do desempenho em testes funcional de indivíduos com IFT com o uso da bandagem elástica ${ }^{7,8}$, bem como, uma tendência de melhora no desempenho esportivo'. Assim, o uso da bandagem tem se difundido cada vez mais em praticantes de atividade física com o intuito de prevenir lesôes, reduzir compensaçóes causadas por disfunçóes crônicas (p.ex. a IFT), bem como, aumentar o desempenho.

Uma modalidade de treinamento que o número de praticantes tem crescido consideravelmente no Brasil é o Crossfit ${ }^{\oplus}$. Este tipo de modalidade de treinamento é um programa de condicionamento com rotinas de

\section{Método}

\section{Sujeitos}

Participaram do estudo, 21 jovens com idade de $27,1 \pm 5,8$ anos, índice de massa corporal de 24,5 $\pm 1,7 \mathrm{~kg} \cdot \mathrm{m}^{-2}$, frequência de treinos semanais de 4,9 $\pm 0,9$ dias e tempo de prática de Crossfit $^{\oplus}$ de 23,9 $\pm 10,7$ meses. Os critérios de elegibilidade foram: praticar Crossfit ${ }^{\oplus}$ regularmente, pelo menos três vezes/semana, durante uma hora por dia, há pelo menos três meses, não apresentar lesão em membro inferior nos três meses anteriores ao estudo, não apresentar instabilidade mecânica articular (negativo para teste de gaveta e/ou inclinação talar) e não fazer uso regular de órteses ou bandagem mecânica ou rígida durante o treinamento. $\mathrm{O}$ tamanho amostral foi calculado com base nos dados de média e desvio padrão, obtidos em estudo piloto, considerando como variável de desfecho o desempenho funcional (pontuação no Multiple Hop Test) para isso foi considerado poder de 0,9 , tamanho de efeito $1,5 \mathrm{e}$ alta intensidade, com movimentos de execução rápida e repetitiva, que tem por um dos objetivos, a melhora no desempenho de atividades desportivas ${ }^{10}$. No estudo de Sprey et al. ${ }^{10}$ foi identificado que cerca de $31 \%$ dos praticantes de Crossfit apresentaram algum tipo de lesão durante o treinamento. Assim, considerando que este tipo de treinamento envolve atividades com saltos e a sustentação do corpo em um único membro, o tornozelo pode ser uma articulação afetada por lesóes.

A bandagem elástica é um recurso terapêutico que vem sendo prescrito para prevenção de lesóes, reabilitação de disfunçôes músculo-esqueléticas e aperfeiçoamento atlético. Porém, o efeito deste recurso sobre o controle motor e biomecânica de gestos atléticos, ainda é pouco esclarecido. Assim, faz-se necessário a realização de estudos que identifiquem o efeito deste recurso no sistema neuromuscular e na biomecânica. O objetivo deste estudo foi avaliar o efeito do uso da bandagem elástica na co-contração dos músculos do tornozelo, na cinemática e no desempenho durante testes funcionais em jovens praticantes de Crossfit ${ }^{\oplus}$. A hipótese inicial do presente estudo é de que o uso da bandagem elástica, em decorrência da resistência elástica, propicia redução da aceleração do tornozelo no momento da aterrissagem, bem como, aumenta a co-contração antagonista, devido ao estimulo tátil e sensibilização dos fusos neuromusculares ${ }^{1,2}$.

alfa erro de 5\%. Todos os voluntários assinaram o Termo de Consentimento Livre Esclarecido.

\section{Procedimentos e Instrumentação}

A coleta de dados ocorreu em duas visitas ao ambiente de coleta, com intervalo de 48 horas entre cada sessão. No primeiro dia, os voluntários foram avaliados por meio de uma ficha de avaliação específica, que registrava: dados antropométricos, histórico de entorses, frequência de treino e avaliação da estabilidade mecânica, por meio dos testes ortopédicos de gaveta anterior e inclinação talar $^{11,12}$. Foi determinado o membro dominante para realização dos testes e posicionamento da bandagem elástica ${ }^{13}$.

Em seguida foram realizadas três contraçōes isométricas voluntárias máximas (CIVM's) dos músculos tibial anterior e gastrocnêmio medial. As CIVM's foram realizadas com duração de 
cinco segundos e 30 segundos de repouso entre cada contração, com o posicionamento do segmento de acordo com Kendall et al. ${ }^{14}$, para os músculos tibial anterior e gastrocnêmio medial. Após as CIVM's os voluntários foram familiarizados com o gesto de salto vertical, posteriormente, foi realizado o sorteio quanto à ordem dos testes, com ou sem bandagem (controle). Por fim, os dados eletromiográficos, cinemáticos e desempenho foram coletados nas condições: bandagem elástica e controle, durante salto vertical.

No segundo e último dia, os voluntários realizaram a familiarização dos testes funcionais. Foram realizados os seguintes testes funcionais: Star Excursion Balance Test (SEBT) ${ }^{15}$ e o Multiple Hop Test (MHT) ${ }^{16}$. Posteriormente, os dados eletromiográficos, cinemáticos e desempenho dos testes funcionais foram coletados nas condiçôes: bandagem elástica e controle.

\section{Eletromiografia}

Para a coleta dos sinais eletromiográficos (EMG) foi utilizado um módulo de aquisição de sinais biológicos (Myotrace, Noraxon, Arizona, EUA), ajustado com frequência de amostragem de $1000 \mathrm{~Hz}$, ganho total de 2000 vezes (20 no pré-amplificador e 100 no amplificador) e modo de rejeição comum de $90 \mathrm{dcB}$. Foram utilizados eletrodos descartáveis circulares de $\mathrm{Ag} / \mathrm{Agl}\left(3 \mathrm{M}^{\oplus}\right.$, São Carlos, Brasil), posicionados em configuração bipolar, com área de captação de $1 \mathrm{~cm}^{2}$ e distância entre eletrodos de $2 \mathrm{~cm}$ posicionados sobre os músculos tibial anterior e gastrocnêmio medial de acordo com normas do Surface Electromyography for Non-Invasive Assessment of Muscles $^{17}$. Previamente a colocação dos eletrodos foi realizada tricotomia e limpeza da pele com álcool, para diminuir possíveis interferências na aquisição dos sinais $\mathrm{EMG}^{17}$. $\mathrm{O}$ eletrodo de referência foi posicionado sobre a patela.

\section{Cinemática}

Os dados cinemáticos foram captados por um acelerômetro 3D (Inline, Noraxon, Arizona, EUA) que foi posicionado entre terceiro metatarso e o cuneiforme lateral, visando à captação da aceleração do tornozelo nos eixos: transversal $(\mathrm{X})$ e horizontal $(\mathrm{Z})^{18}$.

\section{Bandagem Elástica}

Previamente ao posicionamento da bandagem elástica (Kinesiosport, Marília, Brasil), foram realizadas tricotomia e higienizaçáo da área. A bandagem elástica foi aplicada por um fisioterapeuta com formação específica no método. Os passos para posicionamento da bandagem elástica são descritos a seguir e foram adaptados do estudo de Kim et al. ${ }^{7}$ :

- Passo 1: A fita foi dividida em três partes: duas extremidades (sem distensão da fita) e uma parte central (com distensão da fita). No primeiro passo, a parte central da fita foi posicionada sobre articulação talocrural, com $50 \%$ da distensão máxima (terapeuta aplicava maior tensão possível sobre a fita e identificava o percentual necessário para aplicação). As extremidades foram posicionadas sobre maléolo medial e maléolo lateral (FIGURA 1).

- Passo 2: Parte central da fita foi posicionada sobre o retropé (fáscia plantar - regiáo do calcâneo), seguindo sentido caudal-cranial, com $50 \%$ da distensão máxima. As extremidades da fita foram posicionadas sobre os maléolos medial e lateral (FIGURA 1).

- Passo 3: Uma das extremidades foi posicionada acima de maléolo medial, onde a parte central da fita se direcionou, posteriormente, ao tendão do calcâneo e, por fim, foi direcionada para região lateral do retropé, com $40 \%$ da distensão máxima, finalizando com o posicionamento da outra extremidade sobre a regiáo dos ossos naviculares (FIGURA 1).

- Passo 4: Uma das extremidades da fita foi posicionada acima do maléolo lateral, a parte central da fita, se direcionou, posteriormente, ao tendáo do calcâneo e, por fim, foi direcionada para regiáo medial do retropé com $40 \%$ da distensão máxima, finalizando com o posicionamento da outra extremidade sobre a região dos osso cuboide (FIGURA 1).

\section{Star Excursion Balance Test}

O teste SEBT deve ser realizado em apoio unipodal e o membro inferior contralateral deve tocar uma única vez a maior distância possível em três direçôes distintas: ântero-medial, medial e póstero medial ${ }^{15}$. De início os voluntários foram familiarizados como recomendado por Hertel et al. ${ }^{15}$. Foram executadas três tentativas 
validas para cada uma das direçôes, com descanso de 10 segundos entre cada uma das direçóes ${ }^{15}$. Entre as condiçóes bandagem elástica e controle foi determinado um intervalo de cinco minutos.

Para a validação das tentativas, o voluntário deveria manter as mãos na cintura, o pé de apoio no chão, não transferir o peso corporal para o pé que deveria alcançar a distância máxima e deveria manter o equilíbrio durante o alcance ${ }^{19}$. A distância máxima alcançada foi mensurada pelo examinador do teste. Para análise, foram consideradas as médias obtidas em cada direção.

\section{Multiple Hop Test}

O MHT é um teste funcional composto por cinco condiçóes que envolvem saltos ${ }^{16}$. Os cinco testes funcionais são:

- Salto 1: teste de salto para frente na distância máxima: Os voluntários realizavam um único salto para frente, a maior distância possível sem perder o equilíbrio e com apenas um membro inferior. A distância máxima alcançada era mensurada pelo aplicador do teste ${ }^{16}$.

- Salto 2: teste de três saltos para atingir a distância máxima. Os voluntários realizavam três saltos consecutivos, com um único membro inferior, para atingir a maior distância possível. A distância máxima alcançada era mensurada pelo examinador ${ }^{16}$.

- Salto 3: teste de salto em seis metros. Nesse teste, os voluntários foram orientados a saltar o mais rápido possível sobre um percurso de seis metros em linha reta, com um único membro inferior. $\mathrm{O}$ tempo total utilizado para realização do teste foi mensurado pelo aplicador do teste ${ }^{16}$.

- Salto 4: teste de cruzar seis metros saltando. Os voluntários foram instruídos a executar saltos em um único membro inferior, cruzando uma linha reta, com largura de $10 \mathrm{~cm}$ e com distância total de seis metros, o mais rápido possível. O tempo total utilizado para realização do teste foi mensurado pelo examinador do teste ${ }^{16}$.

- Salto 5: Teste de saltos com um único membro inferior. Este teste consiste em saltar durante um percurso formado por oito quadrados, sendo quatro deles nivelados, um com um aclive de $15^{\circ}$, e outro, com um declive de $15^{\circ}$, e os demais com uma inclinação lateral de $15^{\circ}$. Os voluntários foram orientados a executar os saltos sobre um único membro inferior em cada quadrado, o mais rápido possível. O teste foi quantificado pelo tempo usado para completar o caminho, e, para sua avaliação, cada quadrado foi delimitado por uma linha que, quando tocada, era considerada como uma falha, sendo adicionado um segundo extra no tempo total do percurso ${ }^{16}$.

Entre as condições bandagem elástica e controle foi determinado um intervalo de cinco minutos. Para todos os testes foram realizadas duas tentativas válidas. As tentativas eram invalidadas caso ocorresse auxílio do membro contralateral durante a aterrissagem ou passos à frente do mesmo membro que aterrissou. Foram utilizadas as médias das tentativas válidas de cada teste.

\section{Salto Vertical}

Durante o teste do salto vertical, os voluntários eram orientados a saltar verticalmente com contra movimento, o mais alto possível, partindo de apoio bipodal, utilizando os braços durante fase de impulsão. Foram realizadas cinco repetiçôes válidas. Caso o voluntário perdesse o equilíbrio, a tentativa era considerada inválida e o teste realizado novamente. Entre as condiçóes bandagem elástica e controle foi determinado um intervalo de cinco minutos.

\section{Análise de dados}

Os dados de eletromiografia e cinemática foram analisados em ambiente Matlab (Mathworks, Natick, EUA). Os sinais EMG foram filtrados por um filtro passa-banda de $20-500 \mathrm{~Hz}$, retificado pelo método de onda inteira e suavizado por um filtro Butterworth passa baixa de $4^{a}$ ordem com frequência de corte de $6 \mathrm{~Hz}$ e normalizado pelas CIVMs.

Para a análise do percentual de co-contração de gastrocnêmio medial e tibial anterior foi utilizado a Equação 1 de Winter et al. ${ }^{20}$.

$$
\% \mathrm{COCON}=2 \mathrm{x} \frac{\text { areacomum } \mathrm{A} \& \mathrm{~B}}{\text { area } \mathrm{A}+\operatorname{area} \mathrm{B}} \times 100 \%
$$

Onde: $\%$ COCON = percentual de cocontração entre os dois músculos antagonistas; área $\mathrm{A}$ = área abaixo do sinal EMG envelopado da curva do músculo $\mathrm{A}$; area $\mathrm{B}$ = área abaixo do sinal EMG envelopado da curva do músculo B; areacomum A $\& \mathrm{~B}=$ área comum de atividade entre dois músculos antagonistas.

O sinal do acelerômetro foi suavizado por um 
filtro Butterworth passa baixa de $4^{\circ}$ ordem com frequência de corte de $6 \mathrm{~Hz}$. Em seguida, foram identificados os picos e mínimos de aceleração em cada eixo. Para a análise dos dados cinemáticos foram considerados apenas os dados correspondentes à fase de aterrissagem dos saltos. A FIGURA 2 apresenta a delimitação das fases de impulso, voo e aterrissagem.

\section{Estatística}

Para a análise estatística foi utilizado o pacote estatístico PASW 21.0 (SPSS inc, Armonk, EUA). Para as comparaçóes das condiçóes foi utilizado o teste: ANOVA one-way medidas repetidas. O nível de significância adotado foi de $\mathrm{p}<0,05$.

FIGURA 1 - Passos para posicionamento da bandagem elástica.

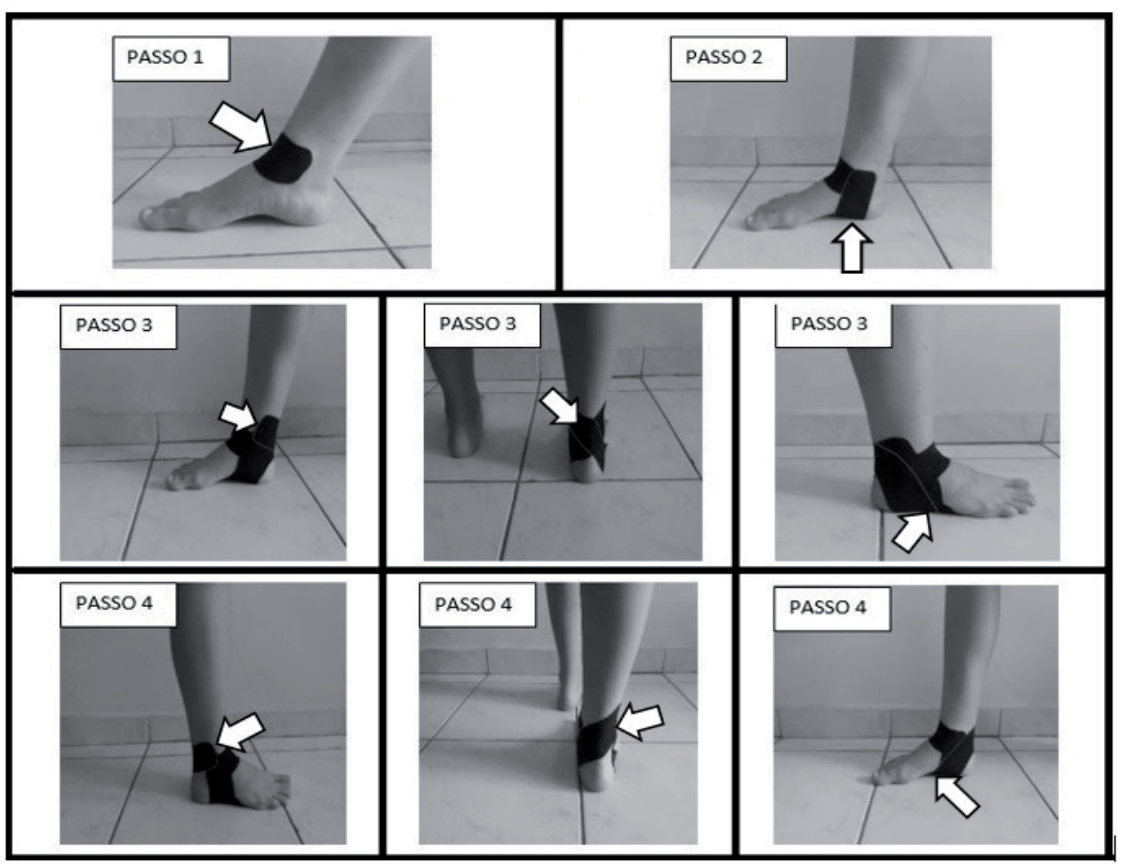

FIGURA 2 - Delimitação das fases de impulso, voo e aterrissagem.

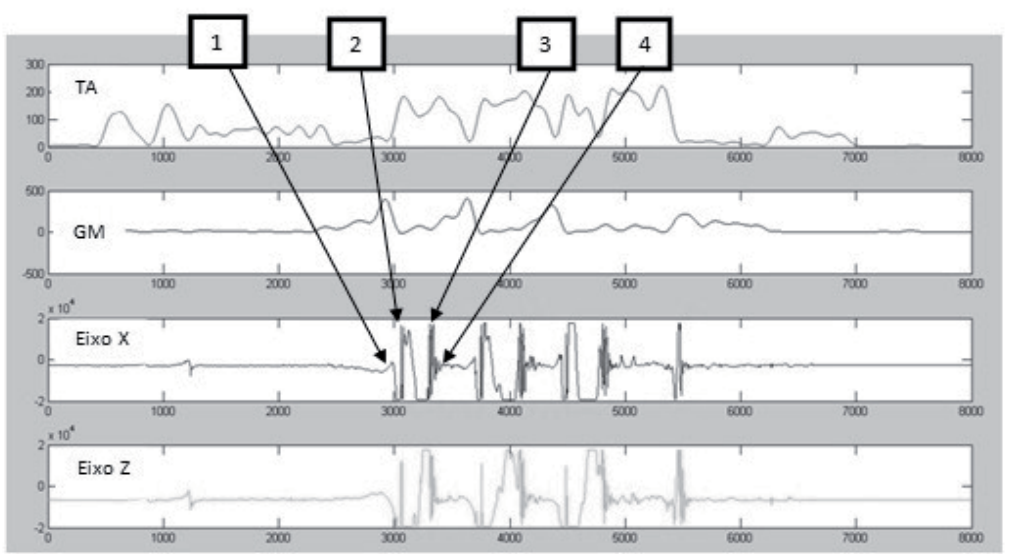




\section{Resultados}

\section{Co-contração}

ANOVA one-way medidas repetidas não encontrou diferença significativa para nenhuma das comparaçóes realizadas de co-contração. Os dados de co-contração são apresentados na TABELA 1.

\section{Cinemática}

ANOVA one-way medidas repetidas não encontrou diferença significativa para nenhuma das comparaçóes cinemáticas realizadas (TABELA 2).

TABELA 1- Dados de co-contração.

\begin{tabular}{lcccc}
\hline & $\begin{array}{c}\text { Bandagem Elástica } \\
(\text { Média } \pm \text { DP })\end{array}$ & $\begin{array}{c}\text { Controle } \\
(\text { Média } \pm \text { DP })\end{array}$ & F & p \\
\hline MHT_S1 & $47,41 \pm 10,3$ & $46,85 \pm 8,3$ & 0,10 & 0,75 \\
MHT_S2 & $46,75 \pm 9,9$ & $46,85 \pm 7,7$ & 0,05 & 0,94 \\
MHT_S3 & $46,63 \pm 10,2$ & $47,27 \pm 7,9$ & 0,22 & 0,64 \\
MHT_S4 & $45,7 \pm 10,0$ & $46,7 \pm 8,8$ & 0,68 & 0,41 \\
MHT_S5 & $46,0 \pm 10,9$ & $45,7 \pm 10,8$ & 0,11 & 0,71 \\
SEBT_Am. & $45,8 \pm 11,2$ & $44,3 \pm 12,2$ & 1,34 & 0,26 \\
SEBT_M & $40,2 \pm 13,5$ & $40,6 \pm 12,3$ & 0,07 & 0,78 \\
SEBT_Pm & $34,4 \pm 12,3$ & $32,6 \pm 11,5$ & 2,00 & 0,17 \\
SV & $38,7 \pm 7,2$ & $38,4 \pm 7,3$ & 0,42 & 0,78 \\
\hline
\end{tabular}

MHT: Multiple Hop Test, S1: Teste de salto para frente na distância máxima,

S2: Teste de três saltos para atingir a distância máxima,

S3: Teste de salto em seis metros,

S4: Teste de cruzar seis metros saltando,

S5: Teste de saltos em um único membro inferior, SEBT: Star Excursion Balance Test,

Am: ântero medial,

M: medial,

Pm: póstero medial,

SV: Salto vertical DP: Desvio Padrão.

TABELA 2 - Comparaçôes cinemáticas.

\begin{tabular}{lcccc}
\hline & $\begin{array}{c}\text { Bandagem Elástica } \\
(\text { Média } \pm \text { DP) }\end{array}$ & $\begin{array}{c}\text { Controle } \\
(\text { Média } \pm \text { DP })\end{array}$ & F & p \\
\hline MHT_S1_AcX & $7,27 \pm 2,12$ & $7,70 \pm 2,68$ & 0,00 & 0,32 \\
MHT_S1_AcZ & $17,78 \pm 2,32$ & $17,85 \pm 3,41$ & 0,10 & 0,26 \\
MHT_S2_AcX & $5,75 \pm 2,49$ & $6,76 \pm 2,73$ & 1,31 & 0,40 \\
MHT_S2_AcZ & $14,66 \pm 2,94$ & $15,43 \pm 3,56$ & 0,70 & 0,12 \\
MHT_S3_AcX & $8,36 \pm 2,85$ & $9,53 \pm 2,65$ & 2,64 & 0,81 \\
MHT_S3_AcZ & $14,42 \pm 3,55$ & $14,66 \pm 3,38$ & 0,05 & 0,52 \\
MHT_S4_AcX & $9,31 \pm 2,54$ & $9,77 \pm 2,12$ & 0,42 & 0,68 \\
MHT_S4_AcZ & $13,93 \pm 2,83$ & $14,16 \pm 2,78$ & 0,17 & 0,67 \\
MHT_S5_AcX & $9,08 \pm 1,64$ & $8,86 \pm 1,70$ & 0,18 & 0,86 \\
MHT_S5_AcZ & $19,94 \pm 19,90$ & $19,86 \pm 2,12$ & 0,03 & 0,54 \\
SV_AcX & $3,99 \pm 2,16$ & $3,47 \pm 2,48$ & 0,38 & 0,91 \\
SV_AcZ & $6,27 \pm 3,84$ & $6,13 \pm 4,53$ & 0,01 & \\
\hline
\end{tabular}

MHT: Multiple Hop Test, S1: Teste de salto para frente na distância máxima,

S2: Teste de três saltos para atingir a distância máxima,

S3: Teste de salto em seis metros,

S4: Teste de cruzar seis metros saltando,

S5: Teste de saltos em um único membro inferior, SV: Salto vertical,

Mud_dir: Mudança de direção,

AcX: Delta entre o valor máximo e mínimo obtidos no eixo $X$ do acelerômetro - flexão plantar,

AcZ: Delta entre os valores máximo e mínimo obtidos no eixo Z do acelerômetro supinação,

DP: Desvio Padrão. 


\section{Desempenho em Testes Funcionais}

Para SEBT, houve aumento da média de alcance máximo de 3,8\% e 5,6\% para as direçóes ântero-medial $(\mathrm{p}=0,001)$ e póstero medial $(p=0,005)$ respectivamente, durante a condição com bandagem elástica. No MHT, houve redução do tempo de execução, em $5,7 \%$ e $7,1 \%$ dos saltos $4(\mathrm{p}=0,018)$ e salto $5(\mathrm{p}=0,009)$ respectivamente, durante condiçáo com bandagem elástica. Os dados são apresentados na TABELA 3.

TABELA 3 - Desempenho em testes funcionais.

MHT: Multiple Hop Test, SEBT: Star Cursion Balance Test,

Am: ântero medial,

M: medial,

Pm: póstero medial,

S1: teste de salto para

frente na distância

máxima,

S2: teste de três saltos para atingir a distância máxima,

S3: teste de salto em seis metros,

S4: teste de cruzar seis metros saltando $\mathrm{e}$

S5: teste de saltos em um único membro inferior,

SV_temp_voo: Tempo de voo do salto vertical. Diferença significativa * $p<0,05$.

\begin{tabular}{lcccc}
\hline & $\begin{array}{c}\text { Bandagem Elástica } \\
(\text { Média } \pm \text { DP) }\end{array}$ & $\begin{array}{c}\text { Controle } \\
(\text { Média } \pm \text { DP })\end{array}$ & F & p \\
\hline SEBT_Am $(\mathrm{cm})$ & $85,01 \pm 6,15$ & $81,84 \pm 6,18$ & 14,81 & $\mathbf{0 , 0 0 1 ^ { * }}$ \\
SEBT_M $(\mathrm{cm})$ & $81,84 \pm 7,39$ & $83,86 \pm 6,10$ & 3,91 & 0,061 \\
SEBT_Pm $(\mathrm{cm})$ & $90,56 \pm 9,15$ & $85,71 \pm 7,49$ & 9,67 & $\mathbf{0 , 0 0 5 ^ { * }}$ \\
MHT_S1 (cm) & $167,55 \pm 29,94$ & $164,88 \pm 33,83$ & 0,43 & 0,515 \\
MHT_S2 (cm) & $472,97 \pm 94,29$ & $467,94 \pm 92,49$ & 0,30 & 0,587 \\
MHT_S3 (s) & $2,34 \pm 0,38$ & $2,36 \pm 0,34$ & 0,15 & 0,695 \\
MHT_S4 (s) & $2,79 \pm 0,54$ & $2,96 \pm 0,61$ & 6,60 & $\mathbf{0 , 0 1 8 ^ { * }}$ \\
MHT_S5 (s) & $3,64 \pm 0,46$ & $3,92 \pm 0,46$ & 8,20 & $\mathbf{0 , 0 0 9 ^ { * }}$ \\
SV_temp_voo & $0,55 \pm 0,10$ & $0,57 \pm 0,08$ & 1,30 & 0,255 \\
\hline
\end{tabular}

\section{Discussão}

Os principais achados deste estudo foram que o uso da bandagem elástica auxiliou no desempenho durante os testes funcionais. De acordo com a teoria que fundamenta a aplicação da bandagem elástica, o uso desta pode estimular mecanoreceptores cutâneos e gerar alteraçóes no controle neuromuscular, tal como aumento da ativação muscular ou co-contraçáo antagonista ${ }^{1,2}$. Entretanto, alguns autores têm sugerido que a melhora do desempenho em variáveis funcionais, como propriocepção e equilíbrio, por meio do uso da bandagem elástica, ocorre em decorrência da maior sensaçâo de segurança provida por esta ${ }^{21,22}$. Dessa forma, os nossos achados discordam da nossa hipótese inicial de que a bandagem elástica poderia ser capaz de alterar o padrão de co-contraçâo dos músculos do tornozelo e a cinemática do tornozelo durante o salto, o que resultaria em melhor desempenho em testes funcionais.

A melhora do desempenho em testes funcionais, encontrado no presente estudo, corrobora parcialmente com os achados de Bicici et al. ${ }^{9}$. Esses achados, relacionados à melhora do desempenho podem, de acordo com SAWKINS et al. ${ }^{23}$, estar relacionados a um efeito placebo, que provem aumento da percepçáo de segurança ao indivíduo.

Em nossa pesquisa não foi possível observar alteraçóes na co-contração entre gastrocnêmio medial e tibial anterior, assim como também não houve alteração na mecânica do movimento. Assim, os resultados do presente estudo corroboram com achados de estudos pregressos que avaliaram o efeito da bandagem elástica, utilizada para estabilização articular e não encontraram alteraçóes eletromiográficas (tempo de onset, ativação e co-contração) ou cinemáticas (deslocamentos angulares e tempo do salto) associadas ao uso desta ${ }^{24,25}$.

Um fator metodológico que pode ter limitado os efeitos da bandagem elástica, foi a distensão aplicada na bandagem. De acordo com Matheus et al. ${ }^{3}$, hipoteticamente, quanto maior forem às distensōes aplicadas na bandagem elástica maiores alteraçôes fisiológicas poderão ocorrer. Assim, a distensão aplicada, no presente estudo, pode náo ter sido o suficiente para gerar alterações eletromiográficas ou cinemáticas. 
Outros fatores como: posicionamento, que pode ter afetado, pois a bandagem não foi colocada sobre os músculos analisados com a direção da força de recuo no sentido da ativação desses músculos, o que pode restringir o efeito tátil desse recurso; o tempo de aplicação; o tipo de material da bandagem elástica; as características da população; e o efeito residual, podem estar relacionados com a não alteração fisiológica. Deste modo é importante destacar que os efeitos da bandagem elástica sobre o sistema neuromuscular e biomecânica, ainda não são completamente compreendidos ${ }^{4-6,26}$.

A aplicação imediata de bandagem elástica não alterou a co-contraçáo e a mecânica do movimento, sugerindo que a bandagem elástica possui limitaçóes como recurso terapêutico. Entretanto, este recurso contribuiu para melhora de desempenho em testes funcionais, o que pode estar relacionado a maior percepção de segurança gerado por este recurso.

\title{
Conflito de interesses
}

Os autores do presente estudo declaram não possuírem conflitos de interesse que enviesassem a realização do mesmo.

\section{Agradecimento}

Agradecimentos ao Crossfit ${ }^{\ominus}$ Marília, pela colaboração e contribuição durante a realização desta pesquisa. Agradecimento a CAPES (Coordenação de Aperfeiçoamento de Pessoal de Nível Superior) pelo apoio financeiro.

\begin{abstract}
Effect of elastic band on functional tests and ankle muscles cocontraction in crossfit athletes

The use of elastic band may generate positive effects on neuromuscular control and movement biomechanics, such as increased joint stability. Thus, the objective of this study was to evaluate the effect of elastic band on ankle muscles cocontraction, kinematics and functional performance in younger Crossfit ${ }^{\circledR}$ athletes. Participate of this study 21 younger adults (age: $27.1 \pm 5.8$ yrs) Crossfit ${ }^{\circledR}$ athletes. In the first day of data collection the follow procedures were performed: maximum isometric contractions for gastrocnemius medialis and anterior tibialis, familiarization and data collection of vertical jump. In the second day of data collection were performed: data collection of functional tests (Multiple Hop Test - MHT; and Star Execution Balance Test - SEBT). All tests were performed in two conditions: with and without the use of elastic band, randomly choose, and an interval of five minutes between each condition. For kinematic analysis was considered the ankle acceleration on the axis: transversal $(X)$ and horizontal (Z), during landing phase of jump. ANOVA one way repeated measures did not found significant difference for co-contraction and kinematics comparison. For the SEBT was found an increment of 3.8 and $5.6 \%$ on the maximum reach for antero-medial $(p=0.001)$ and postero-medial $(p=0.005)$ positions during the condition with elastic band. During MHT there was a reduction of 5.7 and $7.1 \%$ on the time to perform the anterior progression with challenges $(p=0.018$ and $p=0.009)$ in the condition using elastic band. The use of elastic band improves the performance on functional tests in youger Crossfit ${ }^{\circledR}$ athletes, possible it occurred due a placebo effect.
\end{abstract}

KEYWORDS: Kinematic; Electromyography; Physical therapy. 


\section{Referências}

1. Kase K, Hashimoto T, Tomoki O. Development of kinesiotaping perfect manual. Kinesio Taping Ass. 1996;6(10):1178.

2. Martínez-Gramage J, Merino-Ramirez MA, Amer-Cuenca JJ, Lisón JF. Effect of Kinesio Taping on gastrocnemius activity and ankle range of movement during gait in healthy adults: a randomized controlled trial. Phys Ther Sport. 2016;18:56-61.

3. Matheus JPC, Zille RR, Matheus LBG, Lemos TV, Carregaro RL, Shimano AC. Comparison of the mechanical properties of therapeutic elastic tapes used in sports and clinical practice. Phys Ther Sport. 2017;24:74-78.

4. Hsu YH, Chen WY, Lin HC, Wang WT, Shih YF. The effects of taping on scapular kinematics and muscle performance in baseball players with shoulder impingement syndrome. J Electromyography Kinesiol. 2009;19(6):1092-1099.

5. Ho YH, Lin CF, Chang CH, Wu HW. Effect of ankle kinesio taping on vertical jump with run-up and countermovement jump in athletes with ankle functional instability. J Phys Ther Sci. 2015;27(7):2087-2090.

6. Huang CY, Hsieh TH, Lu SC, Su FC. Effect of the Kinesio tape to muscle activity and vertical jump performance in healthy inactive people. Biomedical Eng Online. 2011;10(1):70.

7. Kim BJ, Lee JH, Kim CT, Lee SM. Effects of ankle balance taping with kinesiology tape for a patient with chronic ankle instability. J Phys Ther Sci. 2015;27(7):2405-2406.

8. Lee SM, Lee JH. Effects of ankle eversion taping using kinesiology tape in a patient with ankle inversion sprain. J Phys Ther Sci. 2016;28(2):708-710.

9. Bicici S, Karatas N, Baltaci G. Effect of athletic taping and kinesiotaping ${ }^{\oplus}$ on measurements of functional performance in basketball players with chronic inversion ankle sprains. Inter J Sports Phys Ther. 2012;7(2):154.

10. Sprey JW, Ferreira T, De Lima MV, Duarte Jr A, Jorge PB, Santili C. An epidemiological profile of crossfit athletes in Brazil. Orthopaedic J Sports Med. 2016;4(8):2325967116663706.

11. Cipriano JJ. Manual fotográfico de testes ortopédicos e neurológicos. Artmed Editora, 2016.

12. Clanton TO, Schon LC. Athletic injuries to the soft tissues of the foot and ankle. Surgery Foot Ankle. 1999;2:111453.

13. Hoffman M, Schrader J, Applegate T, Koceja D. Unilateral postural control of the functionally dominant and nondominant extremities of healthy subjects. J AthleticTrain. 1998;33(4):319.

14. Kendall FP, Mccreay EK, Provance PG. Músculos: provas e funções, 4a. ed. Manole, 1995.

15. Hertel J, Braham RA, Hale SA, Olmsted-Kramer LC. Simplifying the star excursion balance test: analyses of subjects with and without chronic ankle instability. J Orthop Sports Phys Ther. 2006;36(3):131-137.

16. Sekir U, Yildiz Y, Hazneci B, Ors F, Aydin T. Effect of isokinetic training on strength, functionality and proprioception in athletes with functional ankle instability. Knee Surgery Sports Traumatol Arthr. 2007;15(5):654-664.

17. Hermens HJ, Freriks B, Disselhorst-Klug C, Rau G. Development of recommendations for SEMG sensors and sensor placement procedures. J Electr Kinesiol. 2000;10(5):361-374.

18. Kapandji AI. Fisiologia articular: esquemas comentados de mecânica humana. Panamericana, 2000.

19. Delahunt E, McGrath A, Doran N, Coughlan GF. Effect of taping on actual and perceived dynamic postural stability in persons with chronic ankle instability. Arch Phys Med Rehab. 2010;91(9):1383-1389.

20. Winter DA. Biomechanics and motor control of human movement. New York: Wiley, 2005.

21. Simon J, Garcia W, Docherty CL. The effect of kinesio tape on force sense in people with functional ankle instability. Clin J Sport Med. 2014;24(4):289-294.

22. De-la-Torre-Domingo C, Alguacil-Diego IM, Molina-Rueda F, López-Román A, Fernández-Carnero J. Effect of kinesiology tape on measurements of balance in subjects with chronic ankle instability: a randomized controlled trial. Arch Phys Med Rehab. 2015;96(12):2169-2175.

23. Sawkins K, Refshauge K, Kilbreath S, Raymond J. The placebo effect of ankle taping in ankle instability. Med Sci Sports Exerc. 2007;39(5): 781-787.

24. De Almeida Lins CA, Neto FL, De Amorim ABC, De Brito Macedo L, Brasileiro JS. Kinesio Taping does not alter neuromuscular performance of femoral quadriceps or lower limb function in healthy subjects: randomized, blind, controlled, clinical trial. Manual Ther. 2013;18(1):41-45.

25. Magalhães I, Bottaro M, Mezzarane RA, Neto FR, Rodrigues BA, Ferreira-Júnior JB, Carregaro RL. Kinesiotaping enhances the rate of force development but not the neuromuscular efficiency of physically active young men. J Electromyography Kinesiol. 2016;28:123-129. 
26. Leong HT, Ng GYF, Fu SN. Effects of scapular taping on the activity onset of scapular muscles and the scapular kinematics in volleyball players with rotator cuff tendinopathy. J Sci Med Sport. 2017;20(6):555-560.

\begin{tabular}{r|c} 
ENDEREÇo & \\
Nise Ribeiro Marques & \\
Universidade do Sagrado Coração & Submetido: 14/ 11/2017 \\
Centro de Ciências da Saúde & Revisado: 18/11/2018 \\
Rua Irmã Arminda, 10-50 & Aceito: 24/05/2021 \\
17011-240- Bauru - SP - Brasil & \\
E-mail: nisermarques@yahoo.com.br & \\
&
\end{tabular}

